\title{
Binding Mode Analysis of Bacillus subtilis Obg with Ribosomal Protein L13 through Computational Docking Study
}

\author{
Yuno Lee, Woo Young Bang, Songmi Kim, Prettina Lazar, Jeong Dong Bahk and Keun Woo Lee*
}

Division of Division of Applied Life Science (BK21 Program), Environmental Biotechnology National Core Research Center (EB-NCRC), Plant Molecular Biology and Biotechnology Research Center (PMBBRC), Gyeongsang National University (GNU), 900 Gazwa-dong, Jinju 660-701, Republic of Korea.

Subject areas:

Bioinformatics/Computational

biology/Molecular modeling

Author contribution: K.W.L., Y.L., W.B. and J.D.B. designed the study; Y.L., W.B. and S.K. carried out literature research; Y.L. and S.K. performed experiments and data analysis; K.W.L., Y.L., W.B., S.K., P.L. and J.D.B. contributed to writing of the paper.

*Correspondence and requests for materials should be addressed to K.W.L. (kwlee@gnu.ac.kr)

Reviewer: Changbong Hyeon, Chung-Ang National University, Republic of Korea. Youngshang Pak Pusan Nat'I Univ., Republic of Korea.

Editor: Sun Choi, Ewha Womans University, Republic of Korea

Received March 23, 2009 ; Accepted March 27, 2009; Published March 30, 2009

Citation: Lee, Y., et al. Binding Mode Analysis of Bacillus subtilis Obg with Ribosomal Protein L13 through Computational Docking Study. IBC 2009, 1(1):3, 1-6.

doi:10.4051/ibc.2009.1.0003

Funding: Yuno Lee, Woo Young Bang, Songmi Kim and Prettina Lazar were recipients of fellowships from the BK21 Programs and this work was supported by grants from the MOST/KOSEF for the Environmenta Biotechnology National Core Research Center (grant \#:R15-2003012-02001-0).

Competing interest: All authors declare no financial or personal conflict that could inappropriately bias their experiments or writing.

Copyright: This article is licensed under a Creative Commons Attribution License, which freely allows to download, reuse, reprint, modify, distribute, and/or copy articles as long as a proper citation is given to the original authors and sources.

\section{SYNOPSIS}

Introduction: GTPases known as translation factor play a vital role as ribosomal subunit assembly chaperone. The bacterial Obg proteins (Spoㅡㅡ-associated GTP-binding protein) belong to the subfamily of P-loop GTPase proteins and now it is considered as one of the new target for antibacterial drug. The majority of bacterial Obgs have been commonly found to be associated with ribosome, implying that these proteins may play a fundamental role in ribosome assembly or maturation. In addition, one of the experimental evidences suggested that Bacillus subtilis Obg (BsObg) protein binds to the $\mathrm{L} 13$ ribosomal protein (BsL13) which is known to be one of the early assembly proteins of the $50 \mathrm{~S}$ ribosomal subunit in Escherichia coli. In order to investigate binding mode between the BsObg and the BsL13, protein-protein docking simulation was carried out after generating 3D structure of the BsL13 structure using homology modeling method.

Materials and Methods: Homology model structure of BsL13 was generated using the EcL13 crystal structure as a template. Protein-protein docking of BsObg protein with ribosomal protein BsL13 was performed by DOT, a macro-molecular docking software, in order to predict a reasonable binding mode. The solvated energy minimization calculation of the docked conformation was carried out to refine the structure.

Results and Discussion: The possible binding conformation of BsL13 along with activated Obg fold in BsObg was predicted by computational docking study. The final structure is obtained from the solvated energy minimization. From the analysis, three important $\mathrm{H}$-bond interactions between the $\mathrm{Obg}$ fold and the $\mathrm{L} 13$ were detected: Obg:Tyr27-L13:Glu32, Obg:Asn76-L13:Glu139, and Obg:Ala136-L13:Glu142. The interaction between the BsObg and BsL13 structures were also analyzed by electrostatic potential calculations to examine the interface surfaces. From the results, the key residues for hydrogen bonding and hydrophobic interaction between the two proteins were predicted. Conclusion and Prospects: In this study, we have focused on the binding mode of the BsObg protein with the ribosomal BsL13 protein. The interaction between the activated Obg and target protein was investigated with protein-protein docking calculations. The binding pattern can be further used as a base for structure-based drug design to find a novel antibacterial drug.

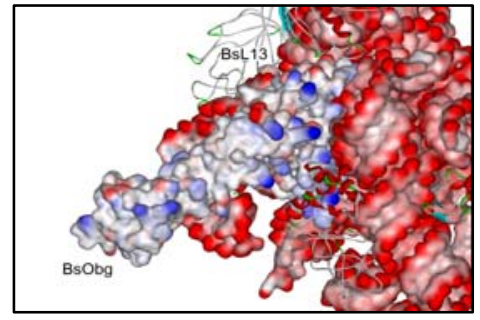

Keywords: Computational docking, Protein-protein docking calculation, DOT, Bacillus subtilis Obg, Ribosomal protein L13 


\section{Introduction}

GTP binding proteins play an essential role in diverse biological processes including signal transduction, protein synthesis, membrane trafficking and cell proliferation (Bourne et al., 1991), and they form a GTPase superclass whose sequence motifs (G1G5) are conserved in almost all organisms ranging from prokaryotes to eukaryotes (Leipe et al., 2002). Notably, Obg subfamily proteins are the GTPases essential for the viability of nearly all bacteria, and they are widely distributed in eukaryotes as well as bacteria. The GTPases such as Era, Obg, YjeQ and YlqF have been widely studied and also known as translation factor. Recently they are also considered as a new target for antibacterial drug (Comartin and Brown, 2006)

Currently, Obg family is one of the GTPase families that can be found in all domains of life. The Obg protein (SpoOB-associated GTP-binding protein) was originally identified in Bacillus subtilis ( $B$. subtilis) as the gene having a GTPase domain located downstream of SpoOB (Trach and Hoch, 1989; Kok et al., 1994; Vidwans et al., 1995), and the B. subtilis Obg (BsObg) has been reported to be essential for the early steps of sporulation and for stress-dependent activation of the $\sigma^{B}$ transcription factor that controls a cellular response to environmental stress (Scott and Haldenwang, 1999; Scott et al., 2000). Besides the BsObg, its bacterial homologs in Caulobacter crescentus, Streptomyces coelicolor, Streptomyces griseus and Vibrio harveyi have been shown to be essential for cell growth, morphological differentiation and DNA replication (Maddock et al., 1997; Okamoto and Ochi, 1998; Slominska et al., 2002). The ObgE, which is an Obg homolog in Escherichia coli (E. coli), has also been reported to be involved in chromosome partitioning, the regulation of DNA replication and DNA repair process as well as cell growth (Kobayashi et al., 2001; Dutkiewicz et al., 2004). Although the basic functions of the Obg subfamily proteins are not clearly proved, the majority of bacterial Obgs have been commonly found to be associated with ribosome (Scott et al., 2000; Lin et al., 2004; Datta et al., 2004; Wout et al., 2004; Sikora et al., 2006; Sato et al., 2005; Tan et al., 2002; Jiang et al., 2006) implying that these proteins may play a role in ribosome assembly or maturation.

Generally, prokaryotes have $70 S$ ribosomes, each consisting of a $30 \mathrm{~S}$ small subunit which includes $16 \mathrm{~S}$ ribosomal RNAs (rRNAs) with S1-S21 ribosomal proteins and a $50 S$ large subunit which contains ribosomal RNAs 5S, 23S with L1-L36 as ribosomal proteins. Ribosomal protein $L 13$ is one of the proteins that forms the large ribosomal subunit. L13 is known to be one of the early assembly proteins of the $50 \mathrm{~S}$ ribosomal subunit in $E$. coli (Ramakrishnan and Moore, 2001; Maguire and Zimmermann, 2001; Chandra Sanyal and Liljas, 2000; Chan et al., 1994). There are some experimental evidences which reveal the specific binding of Obg with ribosomal protein $\mathrm{L} 13$ through an affinity blot assay method (Scott et al., 2000).

The main objective of this study was to investigate the proper binding mode between the BsL13 (B. subtilis L13) and the activated Obg fold in BsObg. In order to achieve the goal, we have constructed the $3 \mathrm{D}$ BsL13 structure using homology modeling to create the same environment with the experimental study. And then protein-protein docking study was followed to find out proper binding conformation between the Obg fold and L13. Finally energy minimization procedure is performed to refine the docked structure. We expect that our result will be helpful for the structure-based drug design for the target protein as an antibacterial agent.

\section{Results and Discussion}

\section{Analysis of the activated BsObg structure}

Based on the two crystal structures of $B$. subtilis and Thermus

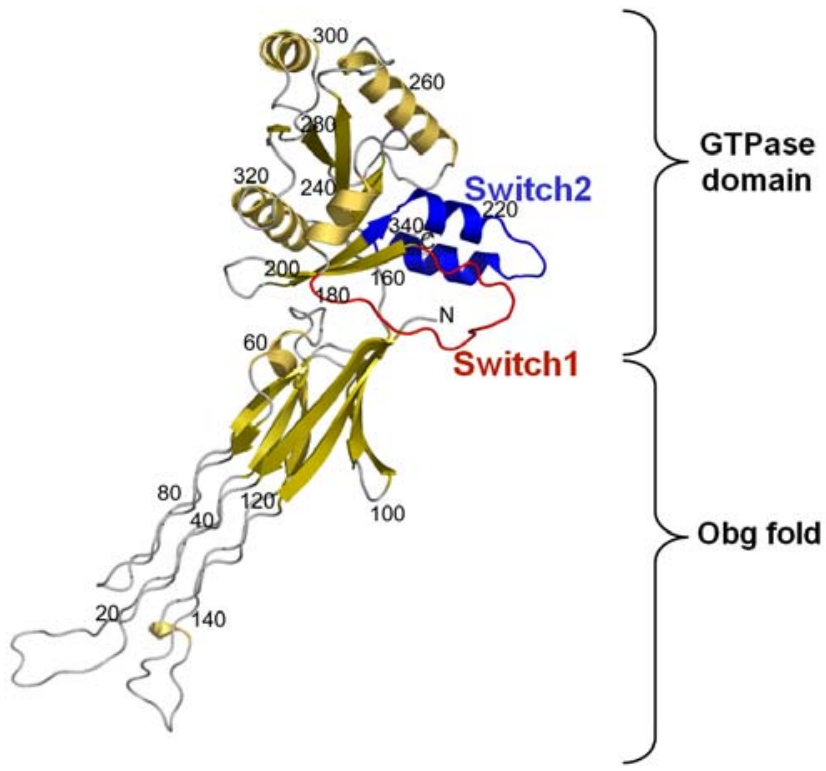

Figure 1. Key element of BsObg structure. Crystal structure of BsObg (PDB ID: 1LNZ) shows Obg fold (residues 1-158) and GTPase domain (residues 159-342) which comprises of switch 1 (red) and switch 2 (blue) elements. C positions were numbered every 20 residues.

thermophilus ( $T$. thermophilus) Obg proteins, structural features of Obg proteins have been elucidated. The Obg proteins contain two equally and highly conserved structural domains, a C-terminal GTP binding domain and an N-terminal glycine-rich domain which is referred as the "Obg fold" (Buglino et al., 2002; Kukimoto-Niino et al., 2004) (Fig. 1). Upon binding of GTP, Obg protein becomes active. It is assumed that such a binding will lead to structural changes within the Obg fold and subsequently it triggers the functional role of the protein. It is already suggested that the Obg fold may be the main platform for protein-protein interaction (Buglino et al., 2002). The orientation of Obg fold may be regulated by guanine nucleotides and further the switch element recognition of GTP-bound configurations can trigger a conformational rearrangement between the domains (Buglino et al., 2002; Kukimoto-Niino et al., 2004). However, the GTP-bound structure of the Obg protein is not determined yet. Thus, we obtained the putative activated form of Obg fold from the $10 \mathrm{~ns}$ molecular dynamics (MD) simulation of the GTP-bound BsObg structure.

The Obg fold has highly conserved positively charged residues in the middle of the loop. This makes the loop highly electropositive in nature (Fig. 2) and gives way for the preferential interaction with the ribosomal protein L13 which has highly electronegative (Fig. 3).

\section{A}
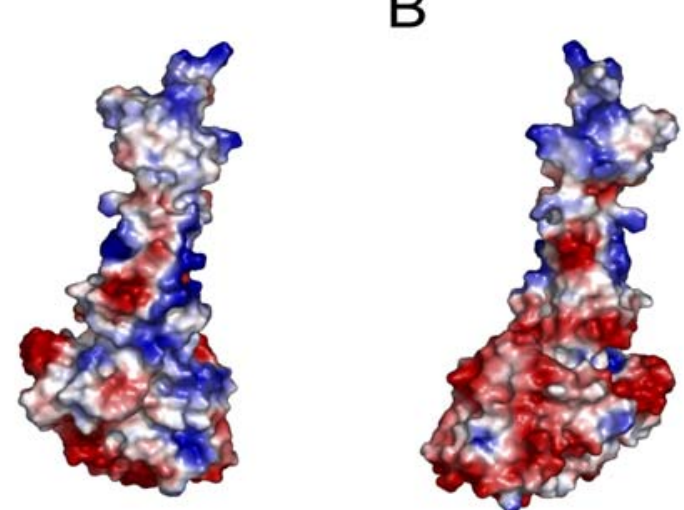

Figure 2. Electrostatic potential (ESP) surface for the BsObg protein. Positive and negative charges are shown as blue and red, respectively: front view (A) and back view (B). 
A

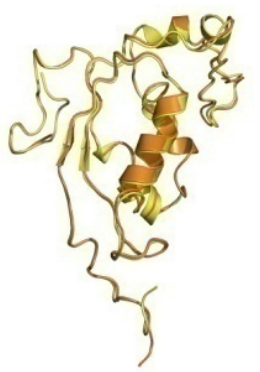

B

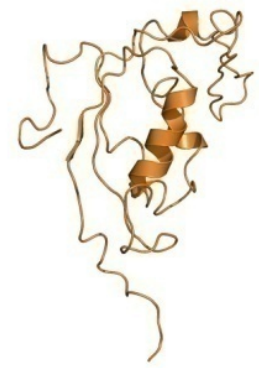

C

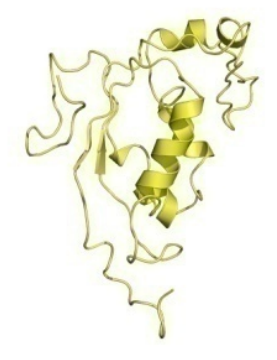

D

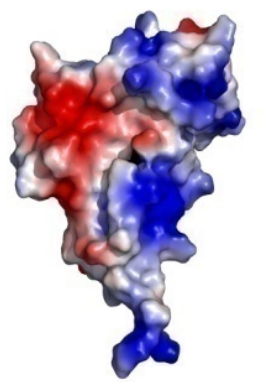

E

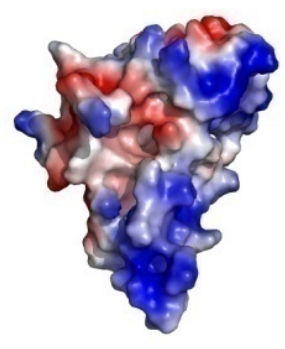

Figure 3. Homology modeled structure of BsL13 with EcL13 as template. Secondary structures of the superimposed EcL13 (orange) and BsL13 (yellow) structures (A) and their individual structure representations EcL13 (B) and BsL13 (C) are represented as cartoons. The electrostatic potential surfaces of the EcL13 (D) and BsL13 (E) homology model are shown.

\section{Building the 3D BsL13 structure by homology modeling}

Homology modeling of BsL13 was carried out using the crystal structure of EcL13 (Escherichia coli L13, PDB ID: 1VS6) as a template (Fig. 3). The EcL13 belongs to the same bacterial source and blastp results showed the best hit with $59 \%$ sequence identity and $75 \%$ similarity. Sequence alignment between EcL13 and BsL13 was performed using ClustalW2 program that gave improved similarity of value $78.7 \%$ with reliable sequence-structure mapping. The reasonable 3D structure of the BsL13 was generated by $M O D E L L E R$, which was then considered as the final homology modeled structure (Fig. 3B). For the model structure, PROCHECK (Schuwirth et al., 2006) predicted $68.5 \%$ of residues lying in the most favored regions, unlike the template protein residues which had about $41.7 \%$ due to low crystallographic resolution. The root mean square deviation (RMSD) between the template structure of EcL13 and the homology model of BsL13 is $1.4 \AA$. Homology) model structure of BsL13 shows similar electrostatic potential (ESP distribution with that of the EcL13 (Fig. 3D and 3E).

3. The L13 structure inside the large ribosomal subunit

Ribosomal protein L13 is one of five proteins required for an early folding of the intermediate of $23 \mathrm{~S}$ rRNA in the large ribosomal subunit maturation (Ramakrishnan and Moore, 2001; Maguire and Zimmermann, 2001; Chandra Sanyal and Liljas, 2000; Chan et al., 1994). The L13 is located at the bottom of the large subunit, near the polypeptide exit site. The L13 can interact with proteins L 3 and L6, and form an extensive network of interactions with 23S rRNA. In the electrostatic potential map, while the positively charged residues of L13 structure are fitted into the 23S rRNA, the negatively charged residues of $L 13$ structure are exposed at outside for the interaction with the Obg fold which is highly electropositive in nature (Fig. 4). It has been already discussed that such electrostatic details are necessary for the ribosome assembly (unpublished data).

\section{Protein-protein docking between Obg and L13 proteins}

The interaction between the activated BsObg fold and the BsL13 protein was investigated with protein-protein docking calculations by DOT 2.0 (Mandell et al., 2001; Adesokan et al., 2003). The analysis of the DOT results was focused on the Obg fold, because the fold is expected to be the contact point with the L13. Among the topranked DOT protein-protein complexes, the best 2000 structure were reevaluated with ACE (pairwise atomic contact energy) score. The ACE is the free energy necessary to replace two residue-water contacts by the corresponding residue-residue and water-water
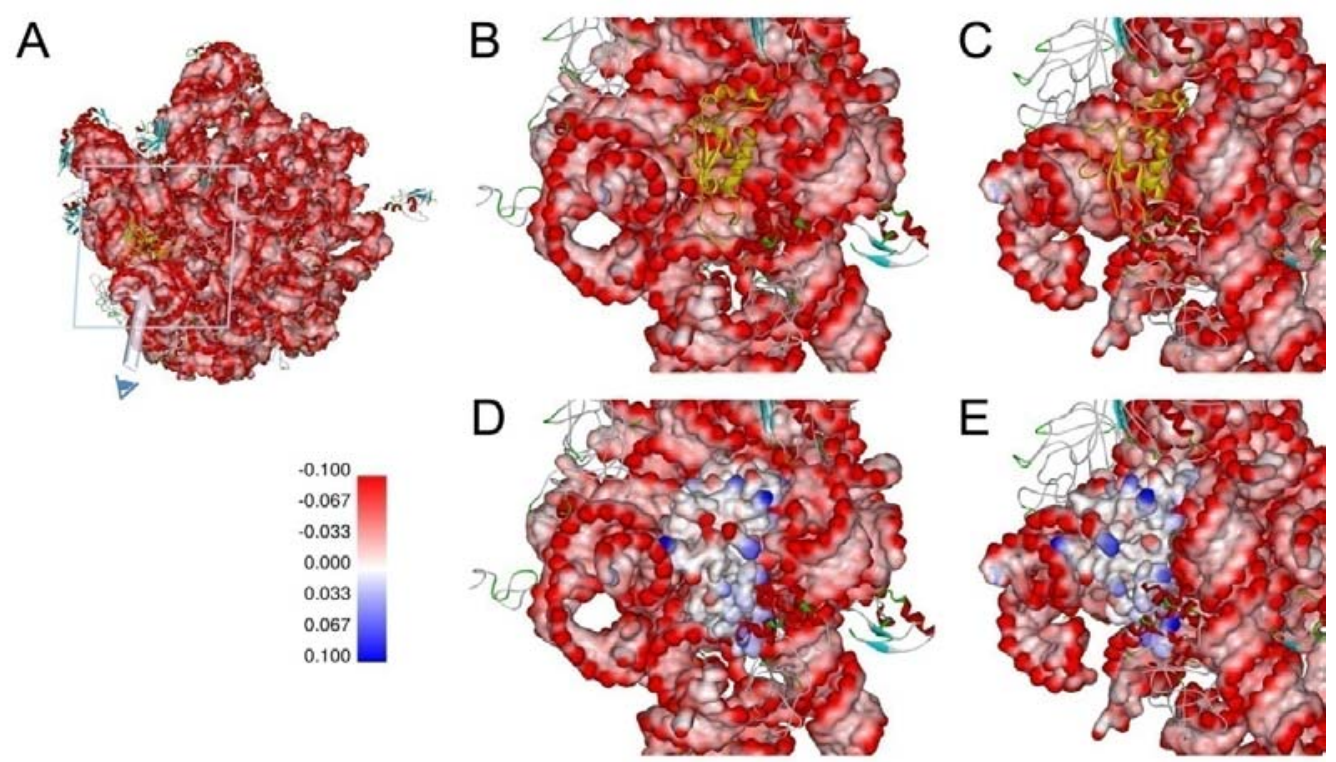

Figure 4. L13 structure in the large ribosomal subunit. (A) Secondary structure of bound L13 represented as cartoons mode (yellow) in $23 S$ rRNA shown as electrostatic surface model (red). The negatively charged residues and the positively charged residues are shown in red and blue, respectively. (B) Clock-wise 90 degree rotation of the zoomed portion of L13 as highlighted by an arrow in panel (A). (C) Side view of panel (B) to clearly show the exposed part. (D) and (E) show the L13 in (B) and (C) as surface model. 
A

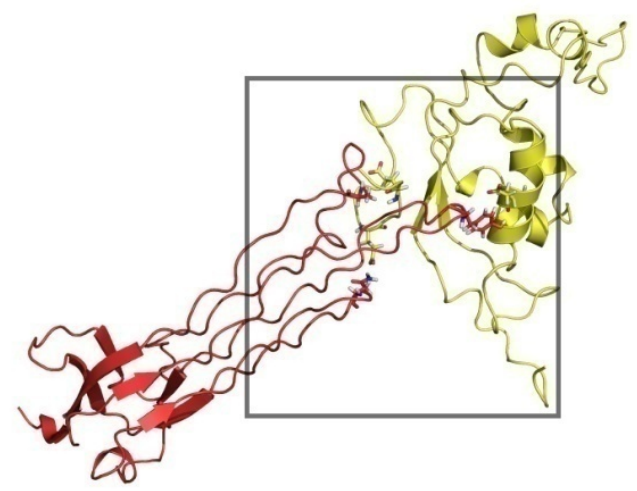

B

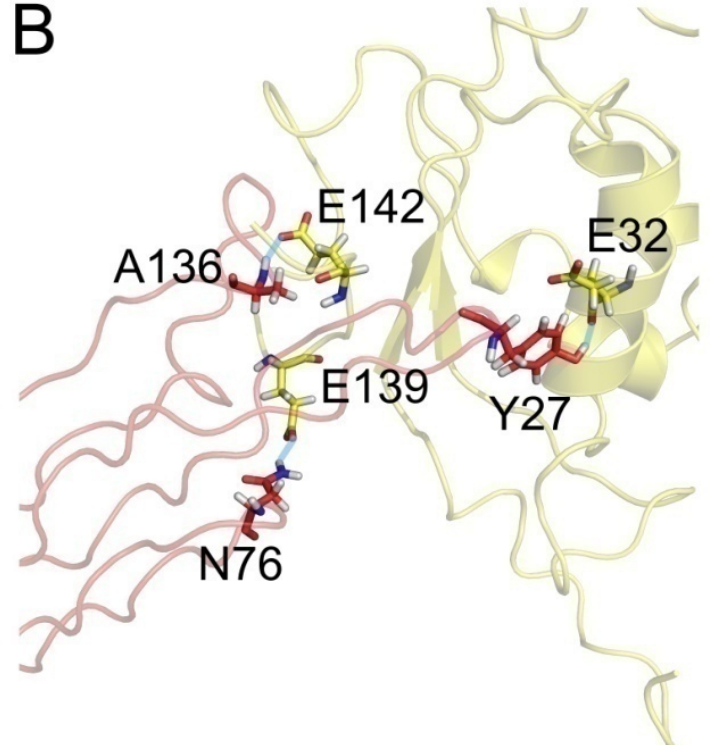

Figure 5. The optimized structure obtained from the protein-protein docking calculation and solvated energy minimization. Binding conformation of the Obg fold (red) with the homology modeled structure of BsL13 (yellow) is represented as cartoon (A) and the detailed view of the key residues (B).

contacts by the corresponding residue-residue and water-water contacts (Chao et al., 1997). The best ranked placement was selected by the energy scores including electrostatic energy, van der Waals energy and the ACE. Finally the optimized structure was obtained after solvated minimization with the best ranked placement (Fig. 5). Three hydrogen bonds and many hydrophobic contacts were detected. Three important $\mathrm{H}$-bond interactions between the Obg and the $L 13$ were predicted from the protein-protein docking study: Obg:Tyr27-L13:Glu32, Obg:Asn76-L13:Glu139, and Obg:Ala136-L13:Glu142 (Fig. 5B).

The differences in the ESP surfaces of the activated Obg fold and the homology model of L13 are clearly depicted by two successive 45 degrees rotations (Fig. 6). The ESP surface examination shows that the positively charged face of the activated Obg fold interacts with the negatively charged face of the homology model of L13. The entire view including the large ribosomal subunit shows that the Obg fold binds only to the exposed surface of the L13, having no contacts with another region of $23 S$ rRNA (Fig. 7). Such an assessment from the docked structure substantiates the interaction between the two proteins.

\section{Conclusion and Prospects}

The bacterial Obg protein belongs to a subfamily of GTP binding proteins. Recently BsObg is recognized as one of the new targets for antibacterial drug. The Obg protein consists of GTP binding domain and a unique Obg fold which is assumed to have an important role in interaction with binding partner. The interaction between the putative activated form of Obg fold and target protein (L13) was further investigated through protein-protein docking calculations. From this, three important $\mathrm{H}$-bond interactions between Obg fold and L13 were predicted: Obg:Tyr27-L13:Glu32, Obg:Asn76-L13:Glu139, and Obg:Ala136-L13:Glu142. Our result can provide more detailed information on the experimental data which show the interaction between the BsObg and the BsL13 (Scott et al., 2000).

Thus from our study, we were able to predict the possible binding conformation of the activated form of Obg fold with L13, which is essential for ribosome assembly. Site-directed mutagenesis experiments can be carried out to validate our results, which can be further used in structure-based drug design (SBDD) for finding

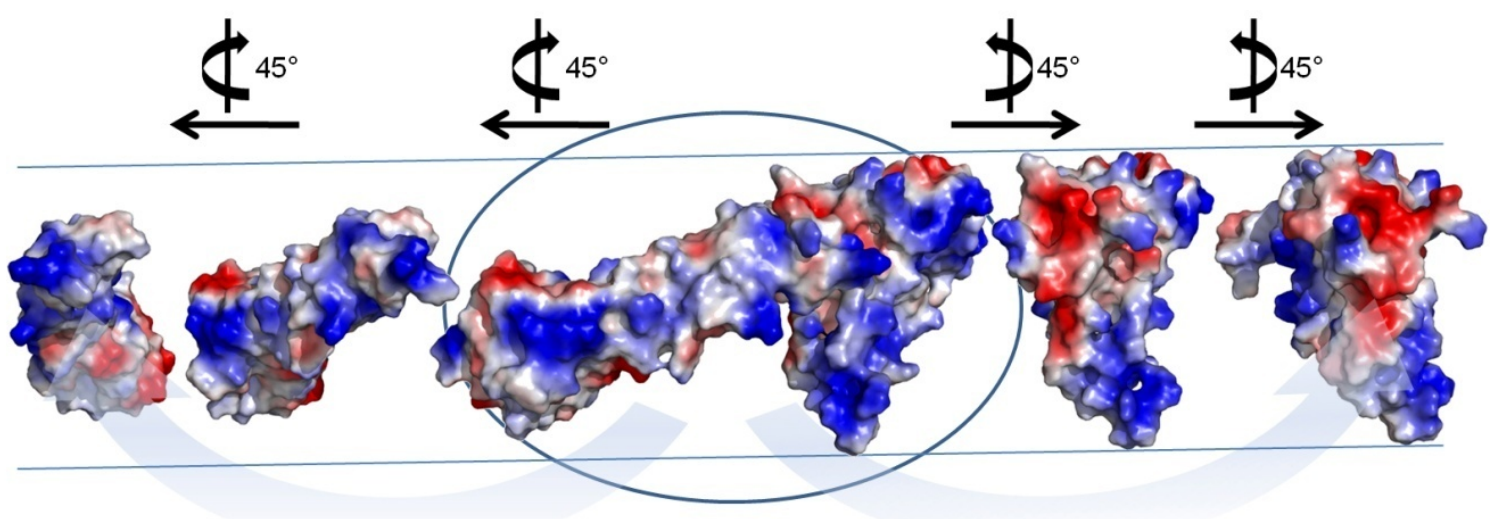

Activated Obg fold

L13

Figure 6. Electrostatic surfaces of the activated Obg fold with the homology model of L13. The activated Obg fold (left) and the homology model of L13 (right) are shown with electrostatic surface and each structure is consecutively rotated by 45 degrees for clarity. The circled shows the docked structure of the activated Obg fold with the L13. 


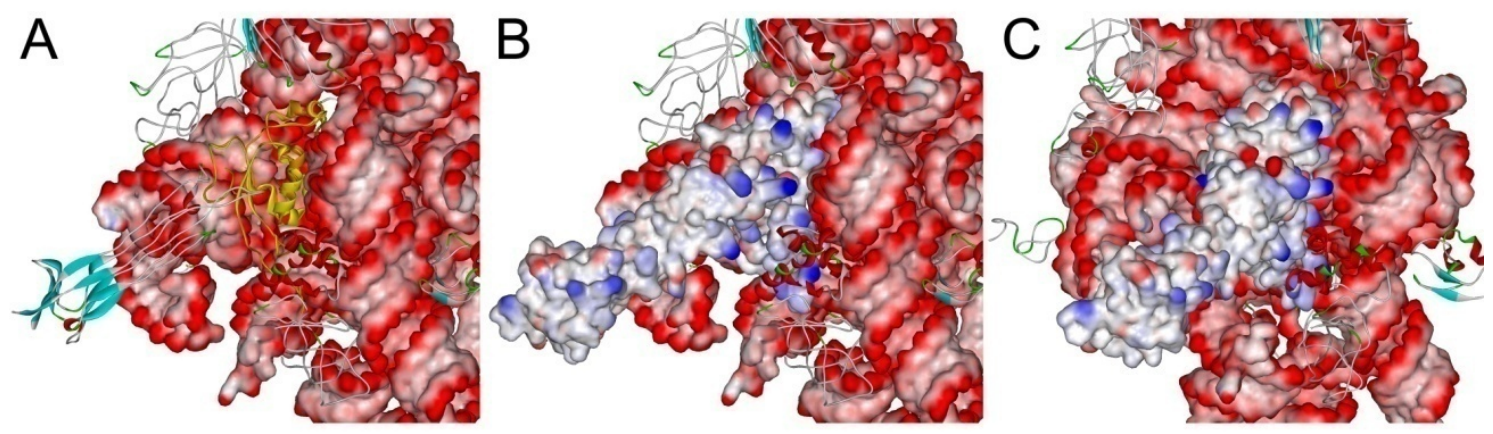

Figure 7. Binding model between the Obg fold and the L13 bound to the large ribosomal subunit. The secondary structure of Obg fold (cyan) bound to the L13 (yellow) in the 23S rRNA (red, electrostatic surface model) is shown as cartoon model ( $A$ ) and surface model (B and C). Panel C shows a different angle view from the view in panel B for clarity. The negatively charged residues and the positively charged residues are shown in red and blue, respectively. (B) Shows Obg fold with L13 as surface model. (C) Front view of figure (B) to clearly show that they are not overlapped.

antibacterial drug.

\section{Materials and Methods}

\section{Preparation of BsObg and BsL13 protein structures}

(1) BsObg protein structure

The 3D structure of activated BsObg was taken from the $10 \mathrm{~ns}$ MD simulation for the GTP bound form of the protein obtained from protein data bank (PDB) structure (PDB ID: 1LNZ) (Mandell et al., 2001). This coordinate of the activated BsObg was selected as the representative structure which was found to be the most closed conformation to the average structure from the last 2 ns trajectories. Polar hydrogen atoms were added to all molecules with the Discovery Studio (Accelrys Inc., San Diego, CA, USA). The GTPase domain with GTP and counterions were removed before the docking simulation to focus on the Obg fold.

\section{(2) Homology modeling of BsL13 structure}

Sequence database search was performed with standard tools, such as blastp and PSI-BLAST to identify the homologue of known structure from the PDB. The coordinates of the EcL13 crystal structure (Escherichia coli L13, PDB ID: 1VS6) (Schuwirth et al., 2006) was used as a template to construct the initial homology model of BsL13 (Bacillus subtilis L13). Sequence alignment between EcL13 and BsL13 was carried out by ClustalW2 program (http://www.ebi.ac.uk/Tools/clustalw2/index.html) (Larkin et al., 2007). The construction of 3D homology model based on the alignment was performed using MODELLER module in Discovery Studio. The final BsL13 model was validated by PROCHECK (Laskowski et al., 1993) along with the evaluation of stereochemical qualities.

\section{Electrostatic potential surface calculation}

The electrostatic potential surfaces for the proteins in Fig. 2, 3, and 6 were calculated by APBS and displayed by PyMOL (Baker et al., 2001) and the ESP surface for the large structure such as the ribosomal subunit in Fig. 4 and 7 were calculated by DelPhi and drawn by Discovery Studio. The dielectric constants of 80 and 2 were used for the solvent and the protein, respectively and the ESP surface for the contours from $-1 \mathrm{kT} / \mathrm{e}$ (red) to $+1 \mathrm{kT} / \mathrm{e}$ (blue) were visualized.

\section{Protein-protein docking calculation by DOT}

DOT (Daughter of Turnip) is a macromolecule-macromolecule docking program that provides a complete search of all orientations between two rigid molecules by systematic rotation and translation of one molecule about another (Mandell et al., 2001; Adesokan et al., 2003). We used this docking program to find out the probable binding conformation between BsObg and BsL13 considering former molecule as stationary molecule and the later one as moving molecule. Almost 54,000 different translated orientations of BsL13 was obtained about the stationary molecule BsObg which was enclosed in an implicitly solvated point grid of dimension $160 \times 160$ $\times 160$. The application of implicitly solvated electrostatics computed by UHBD (University of Houston Brownian Dynamics) and sampled by $D O T$ resulted in the computation of energies of more than 221 billion configurations for protein docking study. Since DOT program can perform a complete search, long-range as well as short-range interactions can be examined.

\section{Solvated energy minimization}

Energy minimization (EM) is needed to provide flexibility and refinement to the docked structure because the DOT program uses rigid molecules for its calculation. The solvated EM was carried out using the GROMACS program (version 3.3.1) (Berendsen et al., 1995; van der Spoel et al., 2005) with GROMOS87 force field. The initial structure was immersed in an orthorhombic water box $(0.8 \mathrm{~nm}$ thickness) and the net charge was neutralized by the addition of 14 $\mathrm{Cl}^{-}$counterions. Long range electrostatics were handled using the particle mesh Ewald (PME) method (Darden et al., 1993). In a system, protein alone consists of 2,986 atoms and the entire system is made up of 61,537 atoms which includes 19,517 water molecules. The steepest descent EM algorithm was used to remove possible bad contacts from the initial structures until energy convergence reached to $2,000 \mathrm{~kJ} \mathrm{~mol}^{-1} \mathrm{~nm}^{-1}$.

\section{Acknowledgement}

Yuno Lee, Woo-young Bang, Songmi Kim and Prettina Lazar were recipients of fellowships from the BK21 Programs and this work was supported by grants from the MOST/KOSEF for the Environmental Biotechnology National Core Research Center (grant \#:R15-2003-012-02001-0).

\section{References}

Adesokan, A. A., Roberts, V. A., Lee, K. W., Lins, R. D. and Briggs, J. M. (2003). Prediction of HIV-1 Integrase/Viral DNA Interactions in the Catalytic Domain by Fast Molecular Docking. J. Med. Chem. 47, 821-828.

Baker, N. A., Sept, D., Joseph, S., Holst, M. J. and McCammon, J. A. (2001). Electrostatics of nanosystems: application to microtubules and the ribosome. Proc. Natl. Acad. Sci. USA 98, 10037-10041.

Berendsen, H. J. C., van der Spoel, D., van Drunen, R. (1995). GROMACS - a message-passing parallel molecular-dynamics implementation. Comput. Phys. Commun. 91, 43-56.

Bourne, H. R., Sanders, D. A. and Mcrmick, F. (1991). The GTPase superfamily: conserved structure and molecular mechanism. 
Nature 349, 117-127

Bourne, H. R., Sanders, D. A. and Mcrmick, F. (1991). The GTPase superfamily: a conserved switch of diverse cell functions. Nature 348, 125-132.

Buglino, J., Shen, V., Hakimian, P., Lima, C. D. (2002). Structural and biochemical analysis of the Obg GTP binding protein. Structure 10, 1581-1592.

Chan, Y. L., Olvera, J., Gluck, A., Wool, I. G. (1994). A leucine zipper-like motif and a basic region-leucine zipper-like element in rat ribosomal protein L13a. Identification of the tumtransplantation antigen P198. J. Biol. Chem. 269, 5589-5594.

Chandra Sanyal, S., Liljas, A. (2000). The end of the beginning: structural studies of ribosomal proteins. Curr. Opin. Struct. Biol. 10, 633-636.

Chao, Z., George, V., James, L. C., Charles, D. (1997). Determination of atomic desolvation energies from the structures of crystallized proteins. J. Mol. Biol. 267, 707-726.

Comartin, D. J., Brown, E. D. (2006). Non-ribosomal factors in ribosome subunit assembly are emerging targets for new antibacterial drugs. Curr. Opin. Pharmacol. 6, 453-458

Darden, T., York, D., Pedersen, L. (1993). Particle mesh Ewald: an $\mathrm{N}-\log (\mathrm{N})$ method for Ewald sums in large systems. J. Chem. Phys. 98, 10089-10092.

Datta, K., Skidmore, J. M., Pu, K. and Maddock, J. R. (2004). The Caulobacter crescentus GTPase CgtAC is required for progression through the cell cycle and for maintaining $50 \mathrm{~S}$ ribosomal subunit levels. Mol. Microbiol. 54, 1379-1392.

Dutkiewicz, R., Słomińska, M., Węgrzyn, G. and Czyż, A. (2002). Overexpression of the cgtA (yhbZ, obgE) gene, coding for an essential GTP-binding protein, impairs the regulation of chromosomal functions in Escherichia coli. Curr. Microbiol. 45, 440-445.

Jiang, M, Datta, K., Walker, A., Strahler, J., Bagamasbad, P., Andrews, P. C. and Maddock, J. R. (2006). The Escherichia coli GTPase CgtAE is involved in late steps of large ribosome assembly. J. Bacteriol. 188, 6757-6770.

Kobayashi, G., Moriya, S. and Wada, C. (2001). Deficiency of essential GTP-binding protein ObgE in Escherichia coli inhibits chromosome partition. Mol. Microbiol. 41, 1037-1051.

Kok, J., Trach, K. A. and Hoch, J. A. (1994). Effects on Bacillus subtilis of a conditional lethal mutation in the essential GTPbinding protein Obg. J. Bacteriol. 176, 7155-7160.

Kukimoto-Niino, M., Murayama, K., Inoue, M., Terada, T., Tame, J. R., Kuramitsu, S., Shirouzu, M., Yokoyama, S. (2004). Crystal structure of the GTP-binding protein Obg from Thermus thermophilus HB8. J. Mol. Biol. 337, 761-770.

Larkin, M. A., Blackshields, G., Brown, N. P., Chenna, R., McGettigan, P. A., McWilliam, H., Valentin, F., Wallace, I. M., Wilm, A., Lopez, R., Thompson, J. D., Gibson, T. J., Higgins, D. G. (2007). ClustalW and ClustalX version 2. Bioinformatics 23, 2947-2948.

Laskowski, R. A., MacArthur, M. W., Moss, D. S., Thornton, J. M. (1993). PROCHECK: a program to check the stereochemical quality of protein structures. J. Appl. Crystallogr. 26, 283-291.

Leipe, D. D., Wolf, Y. I., Koonin, E. V. and Aravind, L. (2002). Classification and evolution of P-loop GTPases and related ATPases. J. Mol. Biol. 317, 41-72.

Lin, B., Thayer, D. A. and Maddock, J. R. (2004). The Caulobacter crescentus CgtAC protein cosediments with the free $50 \mathrm{~S}$ ribosomal subunit. J. Bacteriol. 186, 481-489.

Maddock, J., Bhatt, A., Koch, M. and Skidmore, J. (1997). Identification of an essential Caulobacter crescentus gene encoding a member of the Obg family of GTP-binding proteins. J. Bacteriol. 179, 6426-6431.

Maguire, B. A., Zimmermann, R. A. (2001). The ribosome in focus. Cell 104, 813-816

Mandell, J. G., Roberts, V. A., Pique, M. E., Kotlovyi, V., Mitchell, J. C., Nelson, E., Tsigelny, I. and Ten Eyck, L. F. (2001). Protein Docking Using Continuum Electrostatics and Geometric Fit. Prot. Eng. 14, 105-113.

Okamoto, S., and Ochi, K. (1998). An essential GTP-binding protein functions as a regulator for differentiation in Streptomyces coelicolor. Mol. Microbiol. 30, 107-119.

Ramakrishnan, V., Moore, P. B. (2001). Atomic structures at last: the ribosome in 2000. Curr. Opin. Struct. Biol. 11, 144-154.

Sato, A., Kobayashi, G., Hayashi, H., Yoshida, H., Wada, A., Maeda, M., Hiraga, S., Takeyasu, K. and Wada, C. (2005). The GTP binding protein Obg homolog ObgE is involved in ribosome maturation. Genes Cells 10, 393-408.

Schuwirth, B. S., Day, J. M., Hau, C. W., Janssen, G. R., Dahlberg, A. E., Cate, J. H. D., Vila-Sanjurjo, A. (2006). Structural analysis of kasugamycin inhibition of translation. Nat. Struct. Mol. Biol. $13,879-886$.

Scott, J. M., Ju, J., Mitchell, T., and Haldenwang, W. G. (2000). The Bacillus subtilis GTP binding protein Obg and regulators of the $B$ stress response transcription factor cofractionate with ribosomes. J. Bacteriol. 182, 2771-2777.

Scott, J. M. and Haldenwang, W. G. (1999). Obg, an essential GTP binding protein of Bacillus subtilis, is necessary for stress activation of transcription factor B. J. Bacteriol. 181, 46534660.

Sikora, A. E., Zielke, R., Datta, K. and Maddock, J. R. (2006). The Vibrio harveyi GTPase CgtAV is essential and is associated with the 50 S ribosomal subunit. J. Bacteriol. 188, 1205-1210.

Slominska, M., Konopa, G., Wegrzyn, G. and Czyż, A. (2002) Impaired chromosome partitioning and synchronization of DNA replication initiation in an insertional mutant in the Vibrio harveyi cgtA gene coding for a common GTP-binding protein. Biochem J. $362,579-84$

Tan, J., Jakob, U. and Bardwell, J. C. A. (2002). Overexpression of two different GTPases rescues a null mutation in a heat-induced rRNA methyltransferase. J. Bacteriol. 184, 2692-2698.

Trach, K. and Hoch, J. A. (1989). The Bacillus subtilis spo0B stage 0 sporulation operon encodes an essential GTP-binding protein. J. Bacteriol. 171, 1362-1371.

Van der Spoel, D., Lindahl, E., Hess, B., van Buuren, A. R., Apol, E., Meulenhoff, P. J., et al. (2005). Gromacs User Manual version 3.3, http://www.gromacs.org/

Vidwans, S. J., Ireton, K. and Grossman, A. D. (1995). Possible role for the essential GTP-binding protein Obg in regulating the initiation of sporulation in Bacillus subtilis. J. Bacteriol. 177, 3308-3311.

Wout, P., Pu, K., Sullivan, S. M., Reese, V., Zhou, S., Lin, B. and Maddock, J. R. (2004). The Escherichia coli GTPase CgtAE cofractionates with the $50 \mathrm{~S}$ ribosomal subunit and interacts with SpoT, a ppGpp synthetase/hydrolase. J. Bacteriol. 186, 52495257. 